\title{
Pro-apoptotic and anti-proliferative effects of 3,3'-diindolylmethane in nasopharyngeal carcinoma cells via downregulation of telomerase activity
}

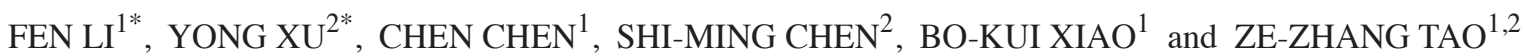 \\ ${ }^{1}$ Research Institute of Otolaryngology-Head and Neck Surgery; ${ }^{2}$ Department of Otolaryngology-Head and Neck Surgery, \\ Renmin Hospital of Wuhan University, Wuhan, Hubei 430060, P.R. China
}

Received August 5, 2014; Accepted April 28, 2015

DOI: $10.3892 / \mathrm{mmr} .2015 .3836$

\begin{abstract}
The pro-apoptotic and anti-proliferative effects of 3,3'-diindolylmethane (DIM) in various tumor cell types have been widely investigated. The underlying mechanisms were suggested to include cell cycle arrest, cell signaling inhibition and downregulation of the androgen receptor. The present study demonstrated that DIM induced apoptosis and inhibited proliferation in nasopharyngeal carcinoma cells by downregulating the activity of telomerase. The nasopharyngeal carcinoma cell line 5-8F was selected for this purpose. A cell counting kit- 8 assay and flow cytometry were performed to detect apoptosis and proliferation of 5-8F cells, respectively, which revealed the pro-apoptotic and anti-proliferative effects of DIM. Telomerase activity was detected using a telomeric repeat amplification protocol assay, which revealed that the telomerase activity was inhibited by DIM in a dose-dependent manner. Reverse transcription polymerase chain reaction was used to detect the mRNA expression levels of human telomerase reverse transcriptase (hTERT) and human telomerase RNA (hTR), and western blot analysis was used to detect the protein expression of hTERT. The results showed that the mRNA and protein expression of hTERT were downregulated in 5-8F cells following treatment with DIM; however, the mRNA expression of hTR remained unchanged, suggesting that hTERT was the target of DIM. To further identify the target, the length of telomeres was continually measured using a telomere length detection
\end{abstract}

Correspondence to: Professor Ze-Zhang Tao, Department of Otolaryngology-Head and Neck Surgery, Renmin Hospital of Wuhan University, 9 Zi-Yang Road, Wuhan, Hubei 430060, P.R. China

E-mail: taozezhang@hotmail.com

*Contributed equally

Key words: 3,3'-diindolylmethane, nasopharyngeal carcinoma, telomerase, human telomerase reverse transcriptase, anti-proliferative, pro-apoptosis kit, revealing that the telomeres were shortened by DIM in an concentration-dependent manner. The present study confirmed that DIM had pro-apoptotic and anti-proliferative effects in nasopharyngeal carcinoma cells by regulating telomerase.

\section{Introduction}

Cruciferous vegetables are plants exerting antitumor activity. 3,3' Diindolylmethane (DIM), which extracted from cruciferous vegetables, induces antiproliferative and proapoptotic effects in a variety of tumor cell types, including nasopharyngeal carcinoma (NPC) cells $(1,2)$. Several underlying mechanisms of the anti tumor effects of DIM have been reported (3-6), however, the DIM regulation of telomerase activity, which is important in NPC, and the associated mechanisms remain to be elucidated. Telomeres are located on the ends of eukaryotic chromosomes, are comprised of G- and $\mathrm{C}$-rich hexanucleotide repeats and protect chromosome ends from recombination, fusion and degradation (7). Cell division is accompanied by a gradual reduction in telomere length. A short telomere triggers the apoptotic program, which, however, can be avoided by the activation of telomerase $(7,8)$. Telomerase is composed of human telomerase RNA (hTR), telomerase-associated protein 1 (TP1) and human telomerase reverse transcriptase (hTERT). As the rate-limiting component of telomerase, hTERT induces the immortalization of a number of cell types in culture (8).

However, whether the effects of DIM may be associated with telomerase activity has remained to be elucidated. Previous studies have investigated the underlying mechanisms of the anti-proliferative and pro-apoptotic effects of DIM and have revealed that cell cycle arrest (9), cell signaling inhibition (10-12) and downregulation of the androgen receptor (13) are involved. The inhibition of telomerase was reported to induce anti-proliferative effects $(14,15)$. The present study hypothesized that telomerase may be important in the anti-tumor mechanism of DIM. Therefore, the effects of DIM on the proliferation and apoptotic rate of NPC cells were assessed. Furthermore, telomerase activity, levels of hTERT and hTR, as well as telomere length were assessed in nasopharyngeal cells treated with DIM. 


\section{Materials and methods}

Cells and culture. Human nasopharyngeal carcinoma (NPC) 5-8F cells were purchased from the Cell Bank of the Chinese Academy of Science (Shanghai, China). The cell line was cultured in the medium RPMI-1640 (HyClone Corp., Logan, UT, USA) supplemented with $10 \%$ fetal bovine serum (Gibco Life Technologies, Carlsbad, CA, USA) and cultured in a humidified incubator at $37^{\circ} \mathrm{C}$ with $5 \% \mathrm{CO}_{2}$.

DIM. DIM (Sigma-Aldrich, St. Louis, MO, USA) was dissolved in dimethylsulfoxide (Sigma-Aldrich) and was diluted to the concentrations of $0,25,50,75$ and $100 \mu \mathrm{M}$ in complete medium (Genom Biotechnology, Hangzhou, China).

Cell proliferation assay. Cells in the logarithmic growth phase were obtained and were seeded into 96-well plates at 2,500 cells/well. Following culturing, cell proliferation was assessed using a cell counting kit-8 (CCK-8; cat. no. C0038; Beyotime Institute of Biotechnology, Shanghai, China) according to manufacturer's instructions. Briefly, $10 \mu \mathrm{l} \mathrm{CCK8}$ solution was added to the culture medium followed by incubation for $1 \mathrm{~h}$. The absorbance was measured at a wavelength of $450 \mathrm{~nm}$ with a reference wavelength of $630 \mathrm{~nm}$.

Flow cytometry. Induction of apoptosis was assessed using flow cytometric analysis. An Annexin V/propidium iodide (PI) apoptosis kit (cat. no. LK-AP101-100; Lianke Biotech Co., Ltd., Hangzhou, China) was used for the detection. The cells were seeded into six-well plates and the next day, the medium was changed to medium containing $0,25,50,75$ and $100 \mu \mathrm{m}$ DIM for up to $24 \mathrm{~h}$, prior to digestion and collection. The samples were stained with Annexin V-fluorescein isothiocyanate (FITC) and PI for $15 \mathrm{~min}$. The cells were analyzed immediately by flow cytometry (FACSCanto II; BD Biosciences, San Jose, CA, USA) using the fluorescence channels FL1 (emission at $525 \mathrm{~nm}$ ) and FL3 (emission at $670 \mathrm{~nm}$ ).

Telomeric repeat amplification protocol (TRAP) assay. For the detection of telomerase activity, a TRAP-polymerase chain reaction (PCR) assay was used (Millipore, Billerica, MA, USA). The primers were as follows: TS, 5'-AATCCGTCGAGCAGAGTT-3' and CX primer, 5'-CCСТTACCСТTACCCTTACCCTAA-3'. Cells $\left(\sim 1 \times 10^{6}\right)$ were washed once with ice-cold wash buffer, re-suspended and centrifuged at $3,000 \times \mathrm{g}$ for $5 \mathrm{~min}$ at $4^{\circ} \mathrm{C}$. The precipitate was homogenized with $40 \mu \mathrm{l}$ cold lysis buffer for $30 \mathrm{~min}$ on ice, followed by centrifugation at $13,000 \mathrm{x}$ g for $30 \mathrm{~min}$ at $4^{\circ} \mathrm{C}$. The supernatants were used for subsequent analyses. The extension reaction was as follows: $5 \mu 1$ 10X TRAP buffer, $1 \mu 1$ deoxyribonucleoside triphosphates, $1 \mu 1$ Taq-DNA polymerase, $1 \mu$ l telomere strand primer, $2 \mu 1$ telomerase extraction, $39 \mu \mathrm{l}$ diethylpyrocarbonate-treated $\mathrm{H}_{2} \mathrm{O}$ and $1 \mu \mathrm{l} \mathrm{CX}$ primer. Prior to PCR, a solution without CX primer was pre-incubated at $23^{\circ} \mathrm{C}$ for $30 \mathrm{~min}$. The PCR for the TRAP assay was performed as follows: $94^{\circ} \mathrm{C}$ for $5 \mathrm{~min}$; 35 cycles of $94^{\circ} \mathrm{C}$ for $30 \mathrm{sec}, 50^{\circ} \mathrm{C}$ for $30 \mathrm{sec}$ and $72^{\circ} \mathrm{C}$ for $90 \mathrm{sec}$; followed by $72^{\circ} \mathrm{C}$ for $10 \mathrm{~min}$. The PCR products were electrophoresed using 10\% SDS-PAGE. The gels were stained with ethidium bromide for $15 \mathrm{~min}$, subsequently scanned and images were captured (Geliamce 200 Gel Imaging system; Perkin Elmer, Waltham, MA, USA).

Reverse transcription (RT)PCR. The total RNA was isolated from $\sim 1 \times 10^{6}$ cells and a $25 \mu 1$ reaction mixture, containing $8.5 \mu \mathrm{l}$ 5X RT buffer, $2 \mu \mathrm{l}$ RT enzyme mix, $2 \mu \mathrm{l}$ primer mix and $12.5 \mu 1$ nuclease-free water, which were contained in the RT-PCR kit (Takara, Dalian, China), were incubated at $65^{\circ} \mathrm{C}$ for $5 \mathrm{~min}$, followed by $42^{\circ} \mathrm{C}$ for $1 \mathrm{~h}$. The cDNA was amplified using the following specific primers: hTERT forward, 5'-CGGAAGAGTGTCTGGAGCAA-3' and reverse, 5'-GGATGAAGCGGAGTCGGA-3'; hTR forward, 5'-TCTAACCCTAACTGAGAAGGGCGTAG-3' and reverse, 5'-GTTTGCTCTAGAATGAACGGGGAAG-3'; Actin forward, 5'-CGTACCACTGGCATCGTGAT-3' and reverse, 5'-GTGTTGGCGTACAGGTCTTTG-3'; GAPDH forward, 5'-GAAGGTGAAGGTCGGAGTCA-3' and reverse, 5'-GGCAGAGATGATGACCCTTT-3'. All primers were designed and synthesized by Sangon Biotechnology, Shanghai, China. The PCR assay was performed as follows: $94^{\circ} \mathrm{C}$ for $5 \mathrm{~min} ; 35$ cycles at $94^{\circ} \mathrm{C}$ for $30 \mathrm{sec}, 60^{\circ} \mathrm{C}$ for $30 \mathrm{sec}$ and $72^{\circ} \mathrm{C}$ for $90 \mathrm{sec}$; followed by $72^{\circ} \mathrm{C}$ for $10 \mathrm{~min}$. The products were subsequently electrophoresed on a $1.5 \%$ agarose gels. The gels were stained with ethidium bromide for $15 \mathrm{~min}$, scanned and images were captured (Geliamce 200 Gel Imaging system; Perkin Elmer).

Western blot analysis. The cells were harvested and lysed with lysate buffer (Guge Biotechnology, Wuhan, China), mixed with protease inhibitor cocktail (Roche, Basel, Switzerland) and phosphorylase inhibitor (Roche) on ice for $15 \mathrm{~min}$, and was subsequently quantified using a BCA kit (Thermo Fisher Scientific, Grand Island, NY, USA). The total protein extracts from each group of cells were resolved by $10 \%$ SDS-PAGE (Guge Biotechnology) and transferred on polyvinylidene fluoride (PVDF) membranes (Millipore, Billerica, MA, USA). Following blocking with 5\% sealing liquid (Guge Biotechnology) for $2 \mathrm{~h}$ at room temperature, the PVDF membranes were washed three times for $15 \mathrm{~min}$ with Tris-buffered saline containing Tween-20 (TBST; Guge Biotechnology) at room temperature and incubated with primary antibody (rabbit anti-hTERT; 1:500; cat. no. sc-7212; Santa Cruz Biotechnology, Santa Cruz, CA, USA) overnight at $4^{\circ} \mathrm{C}$. Following extensive washing with TBST three times for $15 \mathrm{~min}$ each, the membranes were incubated with the secondary antibody, donkey anti-rabbit immunoglobulin $\mathrm{G}$ (cat. no. 926-32213; LI-COR, Lincoln, NE, USA) for $1 \mathrm{~h}$. Following washing three times for $15 \mathrm{~min}$ with TBST at room temperature, the membranes were scanned with the Odyssey CLx Infrared Imaging system (LI-COR).

Telomere length detection. A telomere peptide nucleic acid (PNA) fluorescence in situ hybridization (FISH) kit FITC (cat. no. K5325; Dako Denmark A/S, Glostrup, Denmark) was used for the detection of telomere length. The nuclei were isolated by re-suspending $3 \times 10^{5}$ cells in $2 \%$ Triton X-100 and $0.1 \mathrm{M}$ citric acid buffer (Guge Biotechnology), vortexed and incubated for $10 \mathrm{~min}$ at room temperature. The samples were washed once with phosphate-buffered saline (PBS) and directly subjected to denaturation/hybridization. Denaturation 
$\mathbf{A}$

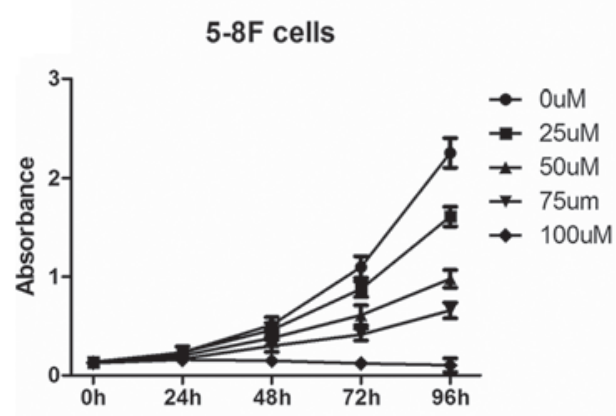

B
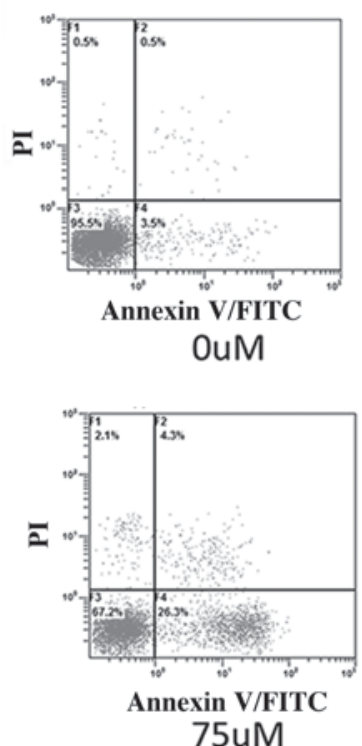

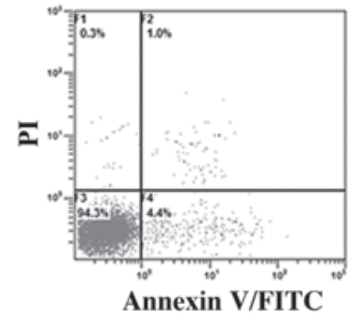

$25 \mathrm{uM}$

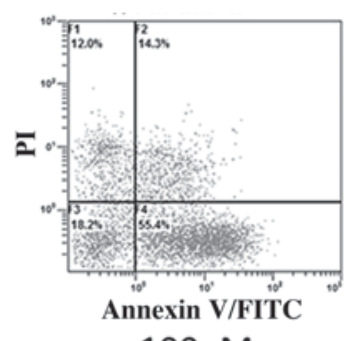

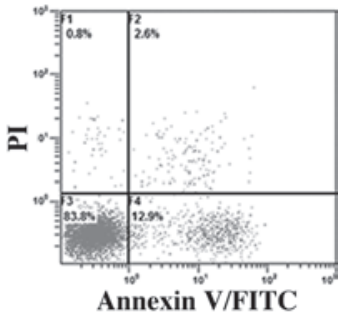

50uM

100uM

Figure 1. Proapoptotic and antiproliferative effects of DIM in the 5-8F NPC cell line. (A) The proliferation rate of the NPC cells treated with DIM at various concentrations was determined. At the time points of $0,24,48,72$ and $96 \mathrm{~h}$, the proliferation ability was detected by CCK- 8 assay. As the concentration of DIM increased, the proliferation ability of the $5-8 \mathrm{~F}$ cells was reduced $(\mathrm{P}<0.05)$. (B) The apoptotic response of the NPC cells treated with DIM at various concentrations was determined. Following $24 \mathrm{~h}$ treatment with DIM, the apoptosis rate of the $5-8 \mathrm{~F}$ cells was detected by flow cytometry. The apoptotic rates were $4.0 \pm 0.4,5.4 \pm 0.6,15.5 \pm 0.5,30.6 \pm 0.5$ and $69.7 \pm 0.6 \%$, respectively. As the concentration of DIM increased, there was an increasing trend in the apoptotic response of the NPC cells $(\mathrm{P}<0.05)$. DIM, 3,3'-diindolylmethane; NPC, nasopharyngeal carcinoma; CCK, cell counting kit; PI, propidium iodide; FITC, fluorescein isothiocyanate.

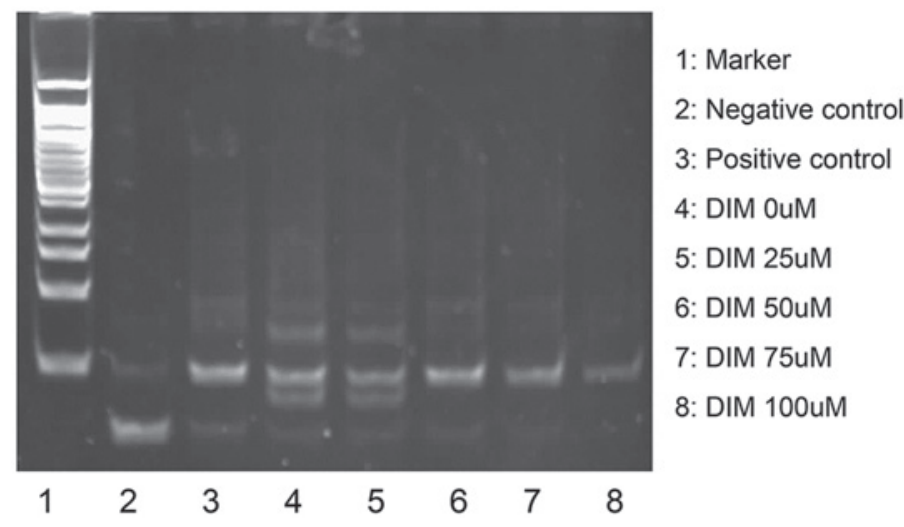

Figure 2. Telomerase activity of 5-8F cells and 5-8F cells treated with DIM. A TRAP assay was performed to detect the telomerase activity. It was demonstrated that the telomerase activity was decreased in a concentration-dependent manner. DIM, 3,3'-diindolylmethane; TRAP, telomeric repeat amplification protocol.

was performed on a thermo block at $80^{\circ} \mathrm{C}$ for $10 \mathrm{~min}$ and the samples were allowed to hybridize at room temperature overnight. The samples were washed with PBS, incubated on a heat block at $40^{\circ} \mathrm{C}$ for $10 \mathrm{~min}$ and subsequently centrifuged at $700 \mathrm{x}$ g for $5 \mathrm{~min}$. The samples were re-suspended in $200 \mathrm{ml}$ DNA staining solution. For DNA counterstaining, the cells were re-suspended in $500 \mu \mathrm{l}$ staining solution for $2 \mathrm{~h}$ prior to acquisition on a FACScan flow cytometer (Becton Dickinson, Franklin Lakes, NJ, USA). The mean telomere fluorescence of the cells was analyzed using Cell Quest software (Becton Dickinson).

Statistical analysis. All statistical analyses were performed using SPSS version 20.0 software (IBM SPSS, Chicago, IL, USA). The values are expressed as the mean \pm standard deviation. A t-test was used to determine the significance.
$\mathrm{P}<0.05$ was considered to indicate a statistically significant difference.

\section{Results}

NPC cell growth is repressed and apoptosis is increased following treatment with DIM. To identify the effects of DIM on NPC cells, the proliferation ability and the apoptotic rate of NPC cells were determined following treatment with DIM. A CCK-8 assay was used to detect the proliferative rate of the NPC cells. The NPC cells were treated with DIM at various concentrations: $0,25,50,75$ and $100 \mu \mathrm{m}$, for five durations: 0 , 24, 48, 72 and $96 \mathrm{~h}$. As the duration increased, the absorbance was increased. In addition, at each time-point, the absorbance decreased as the concentration of DIM increased. Of note, the absorbance at each time-point revealed no change at a 
concentration of $100 \mu \mathrm{M}$, indicating that cells failed to grow substantially (Fig. 1A).

The apoptotic response of NPC cells was detected by flow cytometry. As the concentration of DIM increased, there was an increasing trend in the apoptotic rate of the NPC cells (Fig. 1B).

Telomerase deactivation is involved in the anti-cancer effect of DIM. To identify whether telomerase activity was changed following treatment with DIM, telomerase activity was detected. A TRAP assay was performed to detect the telomerase activity in $5-8 \mathrm{~F}$ cells and $5-8 \mathrm{~F}$ cells treated with DIM at a range of concentrations as mentioned above. The results demonstrated that the telomerase activity was reduced in the DIM-treated cells compared with that in the control 5-8F cells, and the decreasing effect occurred in a concentration-dependent manner (Fig. 2).

Telomerase subunit hTERT is the possible target of DIM. To assess which part of telomerase was the target of DIM, the expression levels of the two components of telomerase, hTERT and hTR, were detected. The mRNA expression levels of hTERT and hTR were detected by RT-PCR, and the protein expression of hTERT was detected by western blot analysis. The results demonstrated that the mRNA and protein expression levels of hTERT were downregulated in the 5-8F cells treated with DIM compared with those in the control 5-8F cells (Fig. 3). The mRNA expression of hTR remained unchanged (Fig. 3), indicating that hTERT was the possible target of DIM in the regulation of the proliferation and apoptosis of NPC cells.

Telomeres are shortened in the NPC cells treated with DIM. To further confirm the functions of DIM with hTERT, the length of telomeres was detected. A telomere length detection kit was used for the detection and the results demonstrated that the telomeres in the $5-8 \mathrm{~F}$ cells treated with DIM were shortened compared with those in the control 5-8F cells in a concentration-dependent manner (Fig. 4). Combined with the finding of apoptosis being induced by DIM in NPC cells, the present study hypothesized that telomere shortening leads to cell death, as indicated by the increased apoptotic rate.

\section{Discussion}

DIM is a tumor-preventive agent and is a natural product present in cruciferous plants. The anti-tumor effect of DIM has been vastly investigated $(2-6,16,17)$. The underlying mechanisms of in the anti-tumor effects were reported in several previous studies which demonstrated that DIM induces apoptosis of tumor cells (1-4), inhibits proliferation $(2,5,6)$, regulates several signaling pathways (2-4), including phosphoinositide 3-kinase, mitogen-activated protein kinase, Akt and nuclear factor- $\kappa \mathrm{B}$, regulates the cell cycle (5), inhibits tumor angiogenesis $(16,17)$, downregulates the androgen receptor (7) and exerts a preventive and curative role in the development and progression of certain types of tumor $(2,5)$.

Proliferation and apoptosis are important hallmark properties of cancer cells (18). Based on this, the present study aimed to investigate the effects of DIM on the proliferation

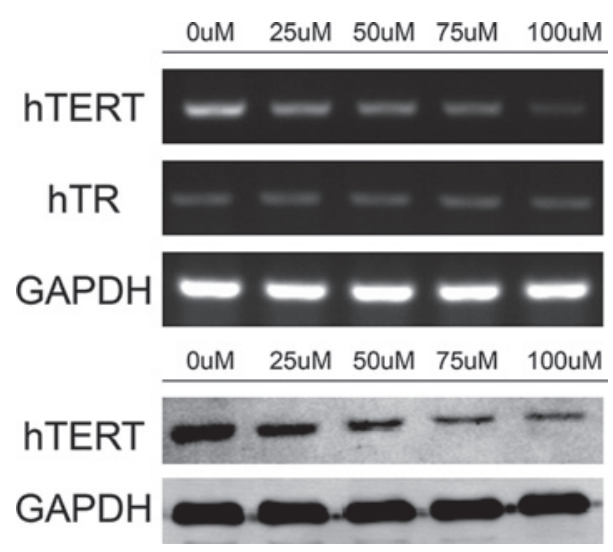

Figure 3. Expression of the telomerase sub-unit of 5-8F cells and 5-8F cells treated with DIM. The cells were treated with $0,25,50,75$ and $100 \mu \mathrm{M}$ DIM for $24 \mathrm{~h}$, and the cells were harvested to detect the protein and mRNA expression levels. The mRNA expression levels of hTERT and hTR were detected by reverse transcription polymerase chain reaction and the protein expression of hTERT was detected by western blot analysis. It was demonstrated that the mRNA and protein expression levels of hTERT were downregulated in the cells treated with DIM compared with those in the untreated 5-8F cells $(\mathrm{P}<0.05)$, while the mRNA expression levels of hTR remained unchanged ( $P>0.05)$. DIM, 3,3'-diindolylmethane; hTERT, human telomerase reverse transcriptase; hTR, human telomerase RNA.

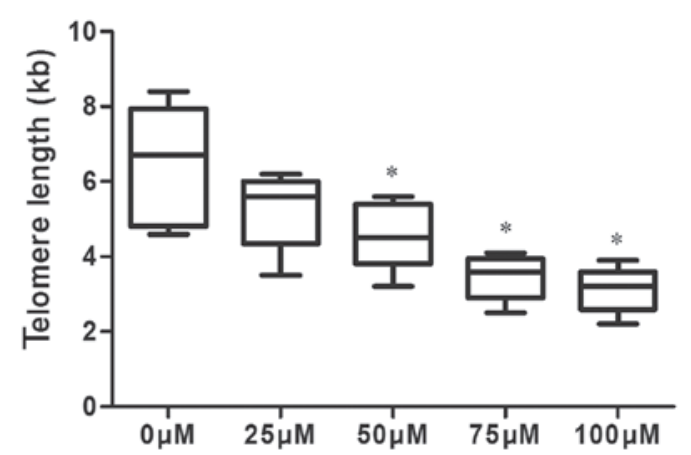

Figure 4. Telomere length in $5-8 \mathrm{~F}$ cells and $5-8 \mathrm{~F}$ cells treated with DIM. The cells were treated with $0,25,50,75$ and $100 \mu \mathrm{M}$ DIM for $24 \mathrm{~h}$, and a telomere length detection kit was used to detect telomere length. The telomere length was graphed as boxes and error bars indicate the mean \pm standard deviation of three replicates. The results were $6.44 \pm 1.6,5.26 \pm 1.0 .4 .58 \pm 0.92,3.46 \pm 0.61$ and $3.12 \pm 0.61$, respectively, and these results indicated that telomeres in cells treated with DIM were shortened compared with those in untreated 5-8F cells. ${ }^{*} \mathrm{P}<0.05$, compared with the $0 \mu \mathrm{M}$ DIM group. DIM, 3,3'-diindolylmethane.

and apoptosis of NPC cells, to identify its effects on telomerase and elucidate the possible underlying mechanism. The proliferation ability of cells with and without DIM treatment was detected using a CCK-8 assay, and the apoptosis of the cells was detected by flow cytometry. The results demonstrated that DIM inhibited proliferation and induced apoptosis of the NPC cells.

Telomerase was first characterized by its ability to elongate and maintain telomere length; however, previous studies on mice and cells have revealed that it also functions in other processes (19-22). The novel functions of telomerase were assessed following the elimination of telomerase activity and several functions were reported, including induction of apoptosis, participation in DNA repair, association with DNA replication protein primase, regulation of DNA damage 
responses, modulation of several signaling pathways and regulation of gene expression.

Telomerase activity was hypothesized to affect changes in proliferation and apoptosis of NPC cells (23); therefore, the present study used the TRAP-PCR assay for the detection of telomerase activity. The results demonstrated that telomerase activity was inhibited following treatment with DIM. Based on these results, the present study aimed to determine which sub-unit of telomerase is a target of DIM. RT-PCR and western blot analyses were performed to detect the mRNA and protein expression levels of hTERT, respectively, and the mRNA expression of hTR. The results demonstrated that the mRNA and protein expression levels of hTERT were downregulated in the DIM-treated cells; however, the mRNA expression of hTR remained unchanged. This suggested that DIM decreased telomerase activity by downregulating the mRNA and protein expression of hTERT.

The expression of hTERT was downregulated in the cells treated with DIM, which confirmed that hTERT was the target of DIM. Inhibition of hTERT by DIM resulted in the inhibition of the telomerase activity. The present study next aimed to determine whether the function of telomerase in this process was associated with telomere length. A FISH assay was used to assess whether the telomere length was affected by DIM, and the results revealed that telomere length was shortened in the DIM-treated cells, suggesting that telomerase inhibition led to decreases in telomere length, inhibited proliferation and directly induced the apoptosis of NPC cells.

Several previous studies have reported that telomerase has a role in the effect of the anti-proliferative and anti-tumor effects of drugs (24-26). Zhao et al (24) revealed that telomerase was inhibited by harmine and the proliferation of MCF-7 cells was decreased. Long-term effects of hTERT inhibition were demonstrated by Qian et al (25), who revealed that SGC-7901 cell proliferation was inhibited by the inhibition of telomerase activity. The present study demonstrated that telomerase activity was inhibited by DIM through the inhibition of hTERT.

Telomerase was identified to be associated with the sensitivity of NPC cells to radiotherapy and chemotherapy $(23,27,28)$. It was hypothesized that DIM may also be involved in the sensitivity to radiotherapy and chemotherapy, and several previous studies confirmed this. Ahmad et al (29) reported that DIM increased the chemotherapeutic sensitivity of prostatic cancer cells, which were multidrug-resistant. Fan et al (30) reported that physiological sub-micromolar concentrations of DIM protected cultured cells against radiation. DIM was thoroughly studied for its functions in cancer therapy and prevention, and indole-3-carbinol, the precursor of DIM, was already approved for clinical use in the treatment of respiratory papillomatosis in the USA (31). It is no exaggeration to suggest that there is a wide prospect for DIM in clinical application; however, further study is required.

In conclusion, the present study reported that the proliferation of NPC cells was inhibited and apoptosis was induced by DIM. NPC cells treated with DIM showed obvious decreases in telomerase activity, and it was speculated that the changes in cell proliferation and apoptosis of NPC cells treated by DIM were associated with telomerase. The mRNA and protein expression of hTERT were downregulated following treatment with DIM, while the expression of hTR mRNA was unaltered, indicating that hTERT was the target of DIM. Furthermore, telomeres were shortened in DIM-treated NPCs, which confirmed that pro-apoptotic and anti-proliferative effects of DIM were mediated through the regulation of telomerase.

\section{Acknowledgements}

The present study was supported by grants from the National Natural Science Foundation of China (no. 81372880), the Independent Research Project of Wuhan University (nos. 2042014kf0184 and 2042014kf0119), the doctoral program of the Higher Education Research Fund (nos. 20130141120093 and 20110141110062) and the Natural Science Foundation of Hubei province (no. 2012FFA045).

\section{References}

1. Wang Q, Tiffen J, Bailey CG, et al: Targeting amino acid transport in metastatic castration-resistant prostate cancer: effects on cell cycle, cell growth and tumor development. J Natl Cancer Inst 105: 1463-1473, 2013.

2. Chen $\mathrm{C}, \mathrm{Chen} \mathrm{SM}, \mathrm{Xu} \mathrm{B}$, et al: In vivo and in vitro study on the role of 3,3'-diindolylmethane in treatment and prevention of nasopharyngeal carcinoma. Carcinogenesis 34: 1815-1821, 2013.

3. Xu Y, Zhang J and Dong WG: Indole-3-carbinol (I3C)-induced apoptosis in nasopharyngeal cancer cells through Fas/FasL and MAPK pathway. Med Oncol 28: 1343-1348, 2011.

4. Banerjee S, Kong D, Wang Z, Bao B, Hillman GG and Sarkar FH: Attenuation of multi-targeted proliferation-linked signaling by 3,3'-diindolylmethane (DIM): from bench to clinic. Mutat Res 728: 47-66, 2011.

5. Chen Z, Tao ZZ, Chen SM, Chen C, Li F and Xiao BK: Indole-3-carbinol inhibits nasopharyngeal carcinoma growth through cell cycle arrest in vivo and in vitro. PloS one 8: e82288, 2013.

6. Kim SJ, Lee JS and Kim SM: 3,3'-Diindolylmethane suppresses growth of human esophageal squamous cancer cells by G1 cell cycle arrest. Oncol Rep 27: 1669-1673, 2012.

7. Blackburn EH: Switching and signaling at the telomere. Cell 106: 661-673, 2001.

8. Shay JW and Wright WE: Senescence and immortalization: role of telomeres and telomerase. Carcinogenesis 26: 867-874, 2005.

9. Tadi K, Chang Y, Ashok BT, et al: 3,3'-Diindolylmethane, a cruciferous vegetable derived synthetic anti-proliferative compound in thyroid disease. Biochem Biophys Res Commun 337: 1019-1025, 2005.

10. Nicastro HL, Firestone GL and Bjeldanes LF: 3,3'-Diindolylmethane rapidly and selectively inhibits hepatocyte growth factor/c-Met signaling in breast cancer cells. J Nutr Biochem 24: 1882-1888, 2013.

11. Zhu J, Li Y, Guan C and Chen Z: Anti-proliferative and pro-apoptotic effects of 3,3'-diindolylmethane in human cervical cancer cells. Oncol Rep 28: 1063-1068, 2012.

12. Li Y, Wang Z, Kong D, et al: Regulation of FOXO3a/beta-catenin/GSK-3beta signaling by 3,3'-diindolylmethane contributes to inhibition of cell proliferation and induction of apoptosis in prostate cancer cells. J Biol Chem 282: 21542-21550, 2007.

13. Bhuiyan MM, Li Y, Banerjee S, et al: Down-regulation of androgen receptor by 3,3'-diindolylmethane contributes to inhibition of cell proliferation and induction of apoptosis in both hormone-sensitive $\mathrm{LNCaP}$ and insensitive $\mathrm{C} 4-2 \mathrm{~B}$ prostate cancer cells. Cancer Res 66: 10064-10072, 2006.

14. Lagah S, Tan IL, Radhakrishnan P, et al: RHPS4 G-quadruplex ligand induces anti-proliferative effects in brain tumor cells. PloS one 9: e86187, 2014.

15. Guo WQ, Li LZ, He ZY, et al: Anti-proliferative effects of Atractylis lancea (Thunb.) DC. via down-regulation of the c-myc/hTERT/telomerase pathway in Hep-G2 cells. Asian Pac J Cancer Prev 14: 6363-6367, 2013.

16. Meng Q, Qi M, Chen DZ, et al: Suppression of breast cancer invasion and migration by indole-3-carbinol: associated with up-regulation of BRCA1 and E-cadherin/catenin complexes. J Mol Med (Berl) 78: 155-165, 2000. 
17. Kong D, Li Y, Wang Z, Banerjee S and Sarkar FH: Inhibition of angiogenesis and invasion by 3,3'-diindolylmethane is mediated by the nuclear factor-kappaB downstream target genes MMP-9 and uPA that regulated bioavailability of vascular endothelial growth factor in prostate cancer. Cancer Res 67: 3310-3319, 2007.

18. Hanahan D and Weinberg RA: Hallmarks of cancer: the next generation. Cell 144: 646-674, 2011.

19. Mukherjee S, Firpo EJ, Wang Y and Roberts JM: Separation of telomerase functions by reverse genetics. Proc Natl Acad Sci USA 108: E1363-E1371, 2011.

20. Martinez P and Blasco MA: Telomeric and extra-telomeric roles for telomerase and the telomere-binding proteins. Nat Rev Cancer 11: 161-176, 2011.

21. Park JI, Venteicher AS, Hong JY, et al: Telomerase modulates Wnt signalling by association with target gene chromatin. Nature 460: 66-72, 2009.

22. Cong Y and Shay JW: Actions of human telomerase beyond telomeres. Cell Res 18: 725-732, 2008.

23. Fujiwara T, Kagawa S and Tazawa H: Synergistic interaction of telomerase-specific oncolytic virotherapy and chemotherapeutic agents for human cancer. Curr Pharm Biotechnol 13: 1809-1816, 2012.

24. Zhao L and Wink M: The beta-carboline alkaloid harmine inhibits telomerase activity of MCF-7 cells by down-regulating hTERT mRNA expression accompanied by an accelerated senescent phenotype. Peer J 1: e174, 2013.
25. Qian X, Cheng J, Chen A, et al: Long-term effects of short hairpin RNA-targeted human telomerase reverse transcriptase on suppression of SGC-7901 cell proliferation by inhibition of telomerase activity. Oncol Rep 19: 575-581, 2008.

26. Zou L, Zhang P, Luo C and Tu Z: shRNA-targeted hTERT suppress cell proliferation of bladder cancer by inhibiting telomerase activity. Cancer Chemother Pharmacol 57: 328-334, 2006.

27. Pang LY and Argyle D: Cancer stem cells and telomerase as potential biomarkers in veterinary oncology. Vet J 185: 15-22, 2010.

28. McCaul JA, Gordon KE, Minty F, Fleming J and Parkinson EK: Telomere dysfunction is related to the intrinsic radio-resistance of human oral cancer cells. Oral Oncol 44: 261-269, 2008.

29. Ahmad A, Sakr WA and Rahman KW: Mechanisms and therapeutic implications of cell death induction by indole compounds. Cancers 3: 2955-2974, 2011.

30. Fan S, Meng Q, Xu J, et al: DIM (3,3'-diindolylmethane) confers protection against ionizing radiation by a unique mechanism. Proc Natl Acad Sci USA 110: 18650-18655, 2013.

31. Wiatrak BJ: Overview of recurrent respiratory papillomatosis. Curr Opin Otolaryngol Head Neck Surg 11: 433-441, 2003. 